\title{
Fair, efficient, and power-optimized spectrum sharing scheme for cognitive radio networks
}

\author{
Saleem Aslam and Kyung Geun Lee
}

\begin{abstract}
The cognitive radio network (CRN) is a promising solution to the problem of spectrum scarcity. To achieve efficient spectrum utilization, cognitive radio requires a robust spectrum sensing and spectrum sharing scheme. Therefore, spectrum sharing scheme plays a key role in achieving the optimal utilization of the available spectrum. The spectrum sharing in CRN is more challenging than traditional wireless network. The main factors besides throughput and fairness which need to be addressed in spectrum sharing of CRN are primary user (PU) activity, transmission power, and variations in the radio environment. In this article, we propose fair, efficient, and poweroptimized (FEPO) spectrum sharing scheme that will incorporate all critical factors mentioned above to maximize the spectrum utilization. Simulation results show that FEPO scheme outperforms in terms of transmission power by reducing the number of retransmissions and guarantees required level of throughput and fairness. Moreover, periodic monitoring helps to reduce the number of collisions with PUs.
\end{abstract}

Keywords: cognitive radio, spectrum sharing, primary user arrival activity, licensed user, FEPO

\section{Introduction}

Current static spectrum management schemes allocate fixed spectrum to each existing wireless network. These schemes assign a block of the spectrum band to a particular radio access-network standard, which is further divided for spectrum allocations into individual operators of this access technology. However, in recent years, wireless network technology grows exponentially especially in the domain of low-cost wireless applications that utilize the unlicensed spectrum bands. These growing applications have raised the issue of spectrum scarcity for upcoming wireless services and stirred the researchers to find new techniques for the efficient utilization of the available spectrum. On the other side of the picture, the Federal Communication Commission has reported that existing spectrum utilization is very sparse at any given time and space $[1,2]$ as shown in Figure 1a.

It shows the variations in power spectral density (PSD) across the radio spectrum from 0 to $6 \mathrm{GHz}$. Although there is a dense spectrum utilization from 0 to $2 \mathrm{GHz}$ yet there is a very sporadic spectrum utilization between

\footnotetext{
* Correspondence: kglee@sejong.ac.kr

Department of Information and Communication Engineering, Sejong
} University, Seoul, Republic of Korea

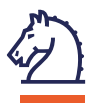

3 and $6 \mathrm{GHz}$. To deal with the problem of the inefficient spectrum utilization, a new concept is evolved called dynamic spectrum access (DSA) or opportunistic spectrum sharing (OSS) [1-3]. The DSA employs cognitive radio $(\mathrm{CR})$, a potential technology to reform the mechanism of spectrum utilization. The DSA architecture consists of two main entities: licensed user (LU) or primary user (PU), which has the legal rights to use the spectrum and CR user or secondary user (SU); CR has temporal rights to utilize the spectrum band of PUs on a negotiation basis. For example, in Figure 1b, there are five PUs and four SUs operating in a cell with single active PU at a given instant.

To avoid harmful interference with PU and to maximize efficiency of the spectrum utilization, CR should periodically sense the radio environment and opportunistically accesses the spectrum hole by dynamically adjusting its transmission parameters like power level, modulation scheme, and coding scheme. There are four major stages of the CR: (1) spectrum sensing, (2) spectrum management, (3) spectrum sharing, and (4) spectrum mobility [3]. The prime objective of CR is the reliable detection and the optimal sharing of spectrum holes among CR users. 


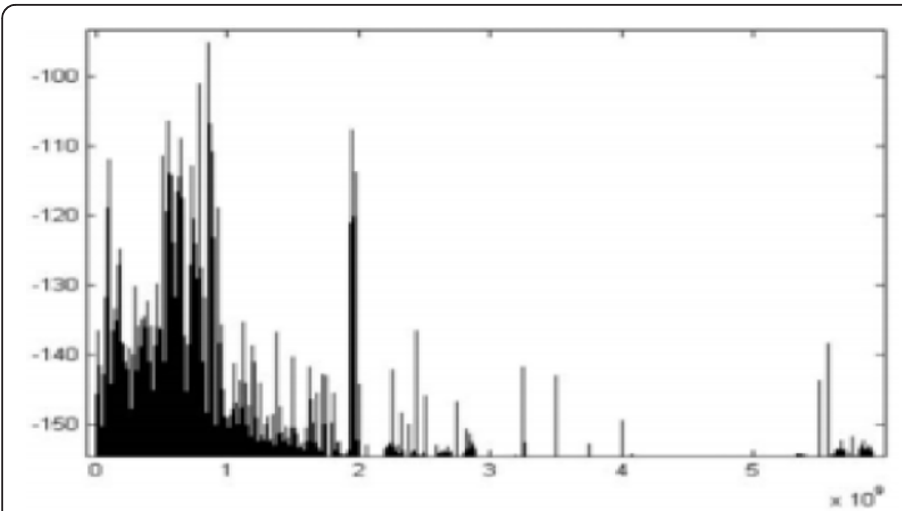

(a)

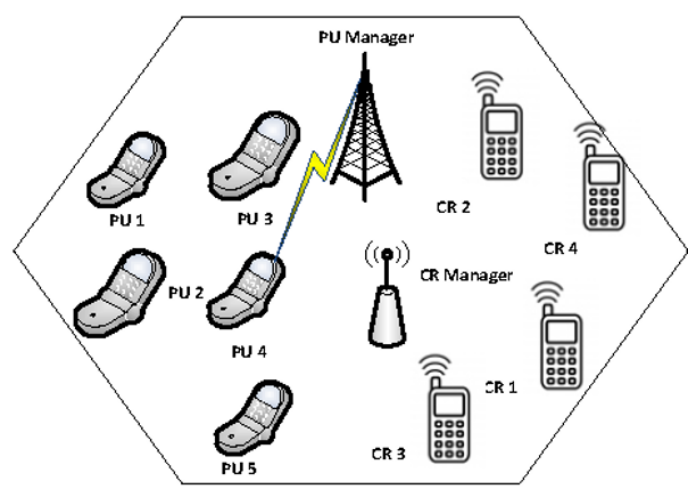

(b)

Figure 1 (a) Spectrum utilization chart 0 to 6 GHz [1]. (b) Operation of CR in similar cell.

Sharing schemes provides a way for spectrum allocation and multiplexing at the data packet level. Moreover, congestion and admission control mechanisms are directly dependent on sharing schemes. Many sharing schemes capable of ensuring required level of QoS in wireless networks have been proposed in the literature. However, these schemes cannot be directly applied to cognitive radio network (CRN) because of the variation in the capacity and quality of wireless channels across space and time and PU arrival activity. Currently, it is an urgent need to develop new spectrum sharing schemes at medium access control (MAC) layer for providing required level of $\mathrm{QoS}$ and operate under tolerable interference limit. Moreover, it is also desirable that the sharing scheme keeps track of the changes occur in the condition and capacity of available wireless channels. Among all other technical issues need to be addressed, spectrum sharing is one of the important issue. In this article, we propose a robust spectrum sharing scheme that will consider all important factors discussed earlier and allocates the available sensed spectrum holes among competing $\mathrm{CR}$ users in an optimal way.

The main contributions of this article are summarized as follows:

(i) We formulate the problem of spectrum sharing in a centralized intra CRN using a slotted structure and considering all relevant metrics and requirements of both SU and network. We provide an in-depth analysis of existing spectrum sharing mechanisms and challenges faced in designing such schemes. This is valuable for future research in this direction.

(ii) We propose a framework for dynamic spectrum sharing in CRN, which incorporates the PU activity as well as changes occurring in the channels due to the fluctuating behavior of the available spectrum in time and apace. To provide a required level of throughput and maximum fairness to the competing CR users, an optimized spectrum sharing strategy is introduced.

(iii) We propose a dynamic framing process at MAC layer, which makes variable size frames depending upon the quality of channel.

(iv) Finally, we compare our proposed scheme with the MMF scheme given in [4] in terms of power consumption to serve the CR users.

The rest of this article is organized as follows. Section 2 briefly presents the previous study related to the spectrum sharing. Section 3 describes the problem formulation process. In Section 4, the impact of PU activity is discussed. The algorithm of the proposed scheme is discussed in Section 5. Simulation results are demonstrated in Section 6. Finally, Section 7 covers the conclusion of the article.

\section{Related study}

Most of the ongoing research in CRN is focused either on physical or MAC layer. The basic aim of the CRN is to provide a way for the efficient utilization of the existing spectrum [5-7]. The CR finds vacant spaces in the licensed band called spectrum holes for opportunistic access [3]. The CRN employs the sensing scheme to detect the presence or absence of the PUs. Spectrum sensing schemes either detector the primary transmitter or receiver. These schemes can also be classified as local or cooperative $[3,8,9]$. In the local spectrum sensing each CR individually decides about the presence of PU, whereas in the cooperative spectrum sensing multiple CR users collectively decide about the presences of PUs on the particular spectrum band. After locating the pool of spectrum holes, these are shared among CR users. In [10], spectrum allocation algorithm is described based on the call request control mechanism. The probability of call blocking is reduced significantly because of the 
call request control mechanism. In [11], another spectrum allocation algorithm is proposed for multi-user OFDM system to maximize the overall capacity of the system. The proposed multi-user algorithm provides better results in terms of capacity and fairness, but it is limited to fully connected networks. A survey of the spectrum sharing scheme in the CRN is presented in [12]. The authors have classified the sharing schemes in three major classes of open, hierarchical, and dynamic exclusive.

The advantages and challenges of each model are also discussed. In [13], the authors present a comprehensive analysis and description on MAC protocols for CRN. It explains the issues related to spectrum sensing, and latest challenges at physical and MAC layers are also discussed in detail. The author categorizes the MAC protocol in three main classes of random access, time slotted, and hybrid protocols. In [14], the authors classify the sharing schemes as centralized or distributive. In the centralized approach, a central entity called a spectrum server or a spectrum broker, which is responsible for sharing the available spectrum band among the CR users while in the distributive method, each $C R$ user participates in the sharing decision. They exchange the information about the sensed spectrum and then collectively share the spectrum among them according to their requirement. Another classification based on architecture is presented in [15] where the sharing schemes are classified as underlay or overlay. The underlay model seems to be the best case as far as the CR operates under the interference level with the PUs but it requires a complex hardware system. In [4,16-18], various centralized spectrum allocation schemes are proposed. In these schemes, each $\mathrm{CR}$ user exchange control-information (CI) with the central server to compete for sensed spectrum holes. The CI contains the sensed information, synchronization information, and power level. Based on this exchanged information, the spectrum server forms an optimal schedule for sharing the spectrum holes among competing CR users. Other random access protocols such as ALOHA and CSMA are presented in [19-21]. The authors propose and simulate a system for the sharing of spectrum holes among CR users, but these techniques are limited to the sharing of a single channel.

In [22] a spectrum sharing scheme based on the interference and power control mechanism is proposed. The author introduces a variable rate and power allocation scheme where each $\mathrm{CR}$ user on different channels has the different amount of transmission power and data rate. The author utilizes multilevel quadrature amplitude modulation to achieve throughput efficiency. The concept of soft sensing information is introduced to get the information about the PU activity and channel state information with respect to the quality of channels. This scheme allocates the available channels under the constraints of bit error rate, and averages transmit power. Although it is an optimal scheme in terms of throughput, but it lacks in providing fairness among CR users that is also an important factor for an optimized sharing scheme.

The sharing schemes in CRN differ from the traditional cellular networks channel sharing techniques because of the capricious nature of the spectrum band in space, time, and quality. This becomes even more challenging if we consider the arrival activity of the PUs as well. Most of the research efforts in CRN are focused to find a way to cater with the interference problem with PUs. There are two main methodologies to deal with the problem of interference with the PUs. In first approach, a predictor forecasts the idle time for the available channels [23-26]. In second approach, interference can be avoided by taking on the fly channel eviction decision. This will degrade the QoS for the SU, but it requires simplified structure as compared to the former approach. In this article, we adopt the latter approach to avoid the interference with the PUs in a centralized intra CRN.

\section{Problem formulation}

In this section, we present the methodology for the formulation of our problem. First, we present the network model, and then proposed the framework of our system. We also present the frame format that we have considered for our system.

\subsection{Network model}

We consider a network with $p=1,2,3, \ldots, P$ PUs and $c=1,2,3,4, \ldots, C$ CR users operating in similar pattern as shown in Figure 1. Each CR user performs sensing operation on $n=1,2,3, \ldots, N$ primary channels of same cell and forward this measurement to the central entity known as CR base station. The primary channel can be modeled as an independent continuous-time Markov process [27]. The transmission on $n$th channel for CR user $c$ using can be modeled using the Markov process as $S_{n}^{c}(t)$. The $S_{n}^{c}(t)=0$ represents the idle state, whereas $S_{n}^{c}(t)=1$ indicates the busy state of channel. The CR can transmit only during the idle state of the channel. We assume the slotted structure for the CR transmission with slot length $\lambda$ as shown in Figure 2 . The slot length $\lambda$ is divided into three subslots. The symbol $\tau$ indicates the sensing time consumed by a particular CR user, $\varepsilon$ represents the channel eviction time period, and $t_{\mathrm{d}}$ represents data transmission period. Mathematically, the slot length is 


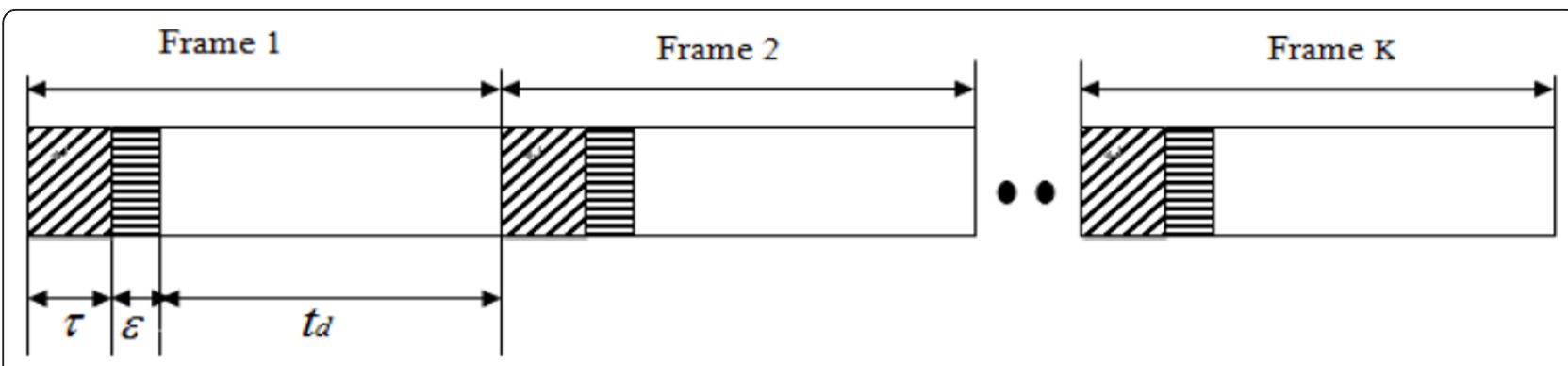

Figure 2 Frame format ( $\tau$ : sensing time, $\varepsilon$ : eviction/switching time and td: actual data transmission time)

$$
\begin{aligned}
& \lambda=\tau+\varepsilon+t_{d} \\
& t_{d}=\lambda-\tau-\varepsilon \\
& \varepsilon<<\tau<<t_{d}
\end{aligned}
$$

We assume that the channel eviction time is very small as compared to the sensing time while the actual data transmission time is significantly larger than the sensing time for a given time slot as shown in Figure 3. Moreover, the allocation is performed after every time slot, and we assume that the environment remains same for the duration of a given time slot.

\subsection{The proposed framework}

Monitoring the PU activity over channels helps significantly to reduce interference with the PUs by vacating/ evicting the channel. Figure 3 represents the proposed framework design for fair, efficient, and power optimized (FEPO) scheme. The general steps can be listed as follows.
Step 1: The PU arrival monitor (PAM) block gathers the statistics about the arrival of PUs on different frequency channels through the spectrum sensing.

Step 2: The PAM analyzes the current spectrum sensing results and sets the value of the channel status flag (csf) for each currently in use frequency channel. If the PU arrives on the same channel, then PAM sets $\operatorname{csf}=1$ for that particular channel.

Step 3: The eviction controller (EC) block observes the csf flags of different channels and preempts/evicts CR users accordingly. For example, if csf of a particular channel is set to 1 , then EC triggers the eviction of $\mathrm{CR}$ user from that channel and at the same time informs the spectrum allocator (SA) about this observation.

Step 4: The SA is the central entity that is responsible for sharing the spectrum among CR users. The SA consists of four elements: (1) channel quality indicator (CQI), (2) user database, (3) a first in first out (FIFO) queue, and (4) a scheduler.

The CQI is responsible for measuring the quality of each unused frequency channel by computing its signal-

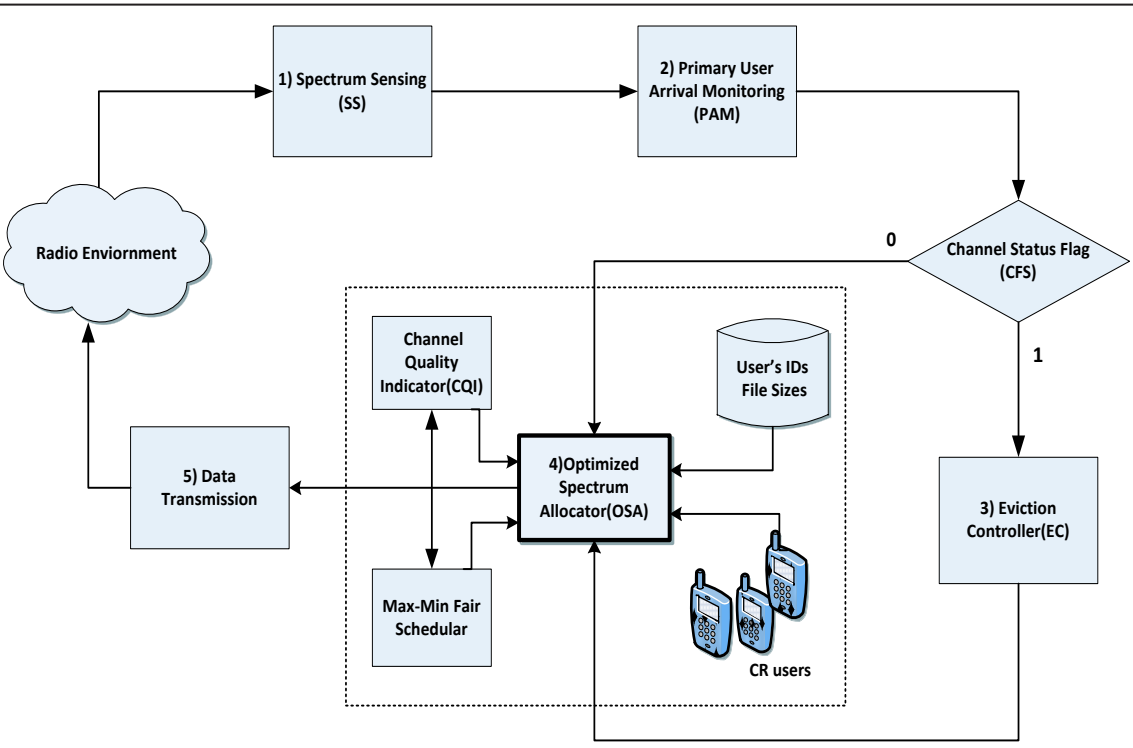

Figure 3 Proposed framework. 
to-interference ratio (SIR). A user database contains the information such as the identifier of CR users, the file size, and the minimum data rate required for each $C R$ user. The FIFO queue maintains the list of CR users competing for channel availability. The spectrum scheduler (SS) forms an optimal schedule by incorporating the observations and calculations from different components within the spectrum allocator with the prime objective of interference avoidance (eviction/silence) with PU and transmission power reduction. We incorporate MMF scheduling algorithm given in [4] to achieve global fairness among CR users. However, if there is a need to vacate a channel on arrival of the PU, then SS will update in-service users with the observation made by EC block.

Step 5: The CR users perform the transmission on the allocated channel and then return to step 1 for sensing.

\section{PU arrival activity}

The CRN utilizes the spectrum band of PUs in an opportunistic manner on the lease basis. From the view point of PUs, it is an important factor that whenever PU needs a spectrum band, CR should vacate the channel to avoid the interference and reduce the number of retransmissions.

Figure 4 represents the on-off activity of PUs on three different channels that we consider for our simulation results. Initially, all three channels are in idle state, i.e., $S_{n}^{c}(t)=0 \forall n$ and available for CR communication. A PU arrives on channel 1 during the slot number 2, the status of channel gets change from idle to busy state, i. e., $S_{n}^{c}(t)=1$ for $n=1$. During sensing interval, CRs sense the arrival activity of $\mathrm{PU}$ and vacate the channel immediately by performing channel eviction/vacation activity with the help of EC block.

\section{Algorithm}

This section describes the algorithm that we have considered for our approach. The details about the different notions and equation are also discussed in this section.

\section{Algorithm: FEPO spectrum sharing scheme}

1. Input: $n_{-} u s e r, n_{-}$ch, $d_{\min }[i]$ and $d_{\max }[i]$ for $(i=1,2$,

3, ..., C) n_user:number of CR user

2. user_serv $\leftarrow 0$, Temp $\leftarrow 0$, csf $\leftarrow 0$; Initialization

3. While (user_ser $==0$ ) do

4. for $n=1$ to $N$

5. If $P U[n]==1$ PUs arrival

6. $\quad \operatorname{csf}[n]=1$;

7. $T$ Temp $=0$;

8. else

9. call CQI; calculate channels quality

10. call max-min fair; call max-min fair

function

11. Temp $=$ ch_data $[n]$;

12. $\quad d_{-} u[n]=d_{-} u[n]-$ Temp; ch_data from maxmin fair

13.

14. If user serve completely

15. $\quad n \_u s e r=n \_u s e r-1$;

16. bring new user;

17. end

18. end

19. if (n_user $==0)$ all user get served

20. user_serv $=1$;

21. end

22. end

23. end

csf channel status flag

dmin minimum data rate requirement of $C R$

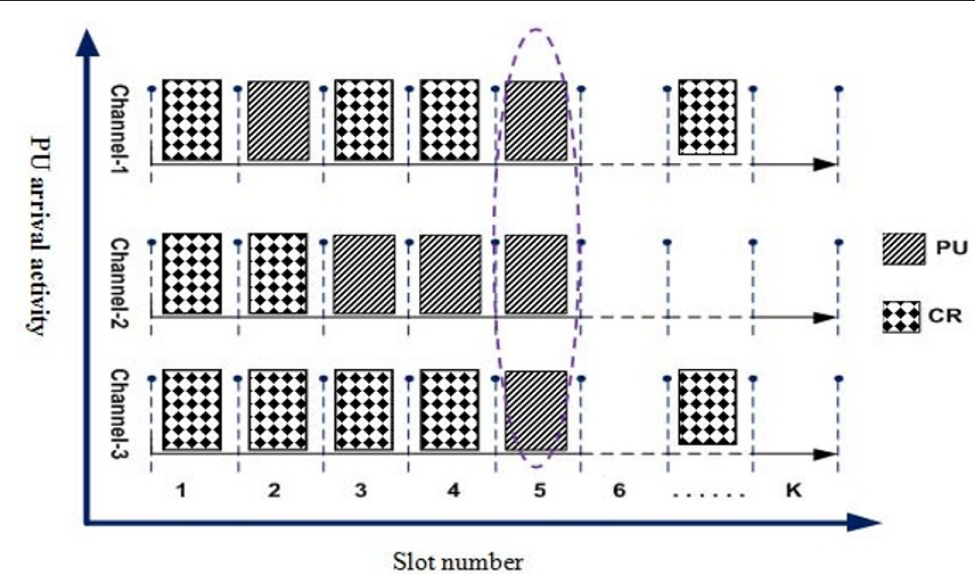

Figure 4 PU's arrival activity 
dmax max data file size of $C R$

$d_{-} u$ user data record variable

$d \_$ch data rate on a channel

In the given algorithm of FEPO, csf represents the channel status flag of $n$th channel in the given time slot. The CQI indicates the channel quality identifier which expresses the quality of channels in terms of SIR. The quality of free channels can be computed by the expression given in [4] as

$$
\Psi_{n}^{m}=\frac{t_{n}^{m} G_{n n} P_{n}}{\sum_{i \neq n} t_{m}^{i} G_{n}^{i} P_{i}+\sigma_{n}^{2}}
$$

where $G_{n n}$ is the channel gain, $P_{n}$ is the power by which CR transmits data on channel $n, t_{n}^{m}$ indicates the on-off pattern of a particular channel, and $\sigma_{n}^{2}$ represents the noise variance. The subscript $m$ indicates the transmission mode. The terms with superscript $i$ represent the effects of the interference from other active $C R$ users on the on the user operating on $n$th channel. As the $C R$ users increase in number, this factor gets increase and hence it will decrease the overall SIR ratio. The data rate on the channels can be calculated using the expression presented in [4] as

$$
d_{n}^{m}=\log \left(1+\Psi_{n}^{m}\right)
$$

where $d$ indicates the capacity of the channel $n$ under certain transmission mode $m$. For the simplicity, we consider the transmission mode in which all the available channels are in active state in a given time slot. In order to achieve fairness, we incorporate MMF scheme discussed in [4] which allocate the equal data rate to all CR users. The proposed scheme also maximizes the throughput while fulfills the minimum data rate requirement $\left(d_{\text {min }}\right)$ of each CR user.

\section{Results and analysis}

In this section, we quantify the performance of our proposed scheme and present simulation results. The simulation program is implemented in Matlab. Although the simulation results are true for more general cases, yet we perform analysis for some specific case to illustrate our outcomes. Our approach is different from the previous studies in terms of taking into account the sharing of the spectrum inconsistency because of irregular PU activity and the changes occur in the radio environment. Moreover, we compare the performance of our proposed technique with previous study in terms of power consumption for the transmission of the CR user's data file. We also incorporate the dynamic framing process within the SA to make the variable frame size. The parameters used for simulation are mentioned in the Table 1.
Table 1 Simulation parameters

\begin{tabular}{ll}
\hline Parameter & Values \\
\hline Slot length & $1 \mathrm{~s}$ \\
Transmission period & $0.9 \mathrm{~s}$ \\
Sensing period & $0.08 \mathrm{~s}$ \\
Channel eviction period & $0.02 \mathrm{~s}$ \\
Number of channels & 3 \\
CR user pairs & 2 \\
Channel gain & $0-1$ \\
Noise variance & $0.2-0.7$ \\
Minimum data requirement & {$[0.5,0.7,0.8]$} \\
Transmission power & $30 \mathrm{~dB}$ \\
\hline
\end{tabular}

\subsection{Impact of selecting transmission the modes and} variations in the channel condition on data rate

Figure $5 \mathrm{a}$ shows the impact of selecting different transmission modes (TM) on the throughput. The TM describes the on-off pattern of available channels. Here, we consider only three channels with eight possible TMs from 000 to 111 as defined in [4]. For example, for TM 001 channel 3 is the best quality channel and has a data rate of $5.39 \mathrm{kbps}$, whereas channel 2 is poor quality with data rate of 4.17 kbps for TM 010. The data rate reduces significantly when two or more channels are active in a given time slot. This reduction in data rate is because of the co-channel interference among CR users. It can be seen that the co-channel interference is the maximum when all the three channels are active under transmission mode 111, but it provides fairness among CR users. As the CR is utilizing the spectrum band of PU on lease basis and accessing opportunistically, there is a significant variation in the channel condition (data rate) across time and frequency during the transmission in each time slot. Figure $5 \mathrm{~b}$ depicts the variation in the data rate achieved for different time slots. As mentioned earlier, the MMF scheme is used to provide same data rate on all available channels. Hence, we plot the variations only for single radio channel in Figure $5 \mathrm{~b}$.

Initially, the data rate on the given channel is 1.82 kbps but data rate decreases during second and third time slots. This decrease in data rate is because of the poor channel condition. The data rate increases again to $1.85 \mathrm{kbps}$ during time slot number 4 because of the significant improvement in the channel condition as compared to the slot number 3 . The maximum data rate of $1.94 \mathrm{kbps}$ and minimum data rate of $1.2 \mathrm{kbps}$ are achieved during time slot numbers 35 and 44, respectively. Hence, the achievable data rate on a channel depends on its condition.

\subsection{Channel eviction activity, sum rate, and channel sharing pattern}

Figure 6 shows the channel eviction behavior and impact of PU activity on the throughput of CR using 


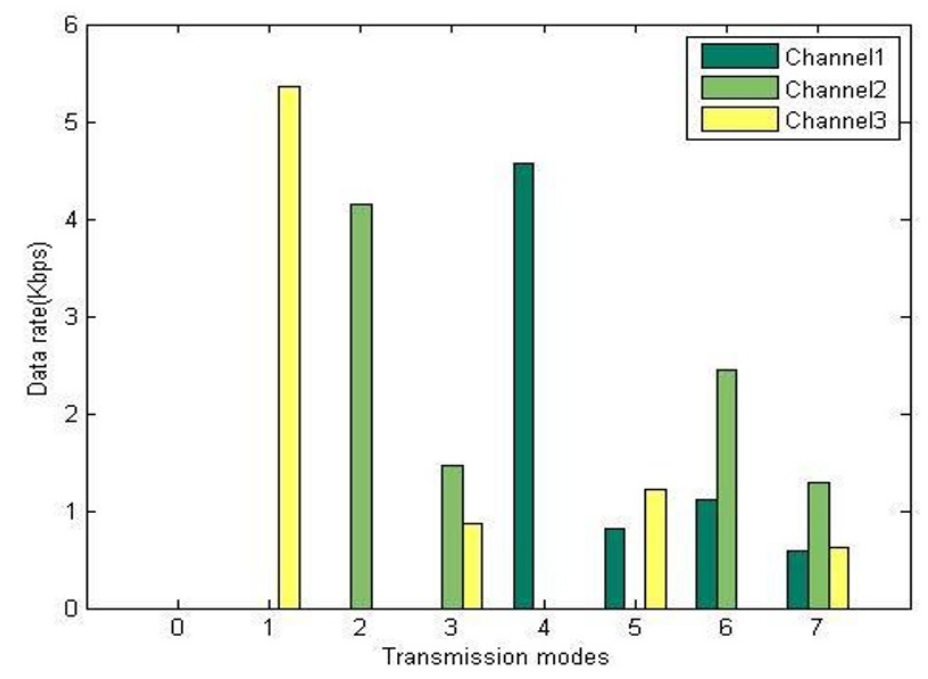

(a)

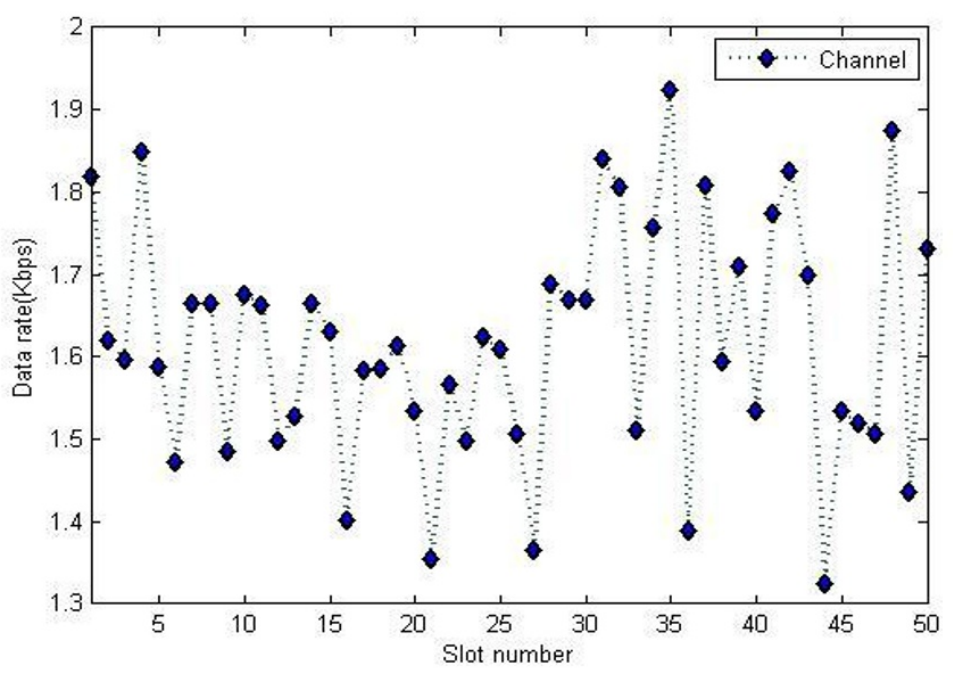

(b)

Figure 5 Impact on data rate. (a) Change in transmission modes [4], (b) Change in nature of spectrum.

the PU arrival pattern depicted in Figure 4. In this case, we consider three CR users with different file sizes of 10,5 , and $10 \mathrm{~kb}$, respectively. Initially, in the first time slot all primary channels are in the idle state. Therefore, these channels can be used for CR communication. In slot number 2, a PU arrives on channel 1. In this case, the PAM block sets the csf $=1$ for channel 1 and inform the SA about the arrival of PU at this time slot. The SA evicts CR from channel 1 by triggering the channel eviction mechanism. This may lead to slight degradation in CR user's throughput operating at the cost of interference avoidance. During time slot numbers 3 and 4, a PU arrives on channel 2, the CR which is currently using channel 2 immediately evicts the channel and switch to channel 1 for its future communication. Lastly, if all the channels are being occupied by PUs then the csf flags of all the channels are set to 1 and SA evicts/preempts all CR users from transmission in order to avoid interference and reduce the power used in retransmissions. This situation happens during slot number 5 as shown in Figure 4. There is a slight variation in the behavior of CR at channels 2 and 3 during time slot number 7 . This variation is due to the early service effect (ESE). The ESE indicates that the remaining file size of $C R$ user is less than the data capacity of channel, and it is serviced fully at the given time slot. The same argument is true for the small data usage on channel 1 during time slot number 8 . 


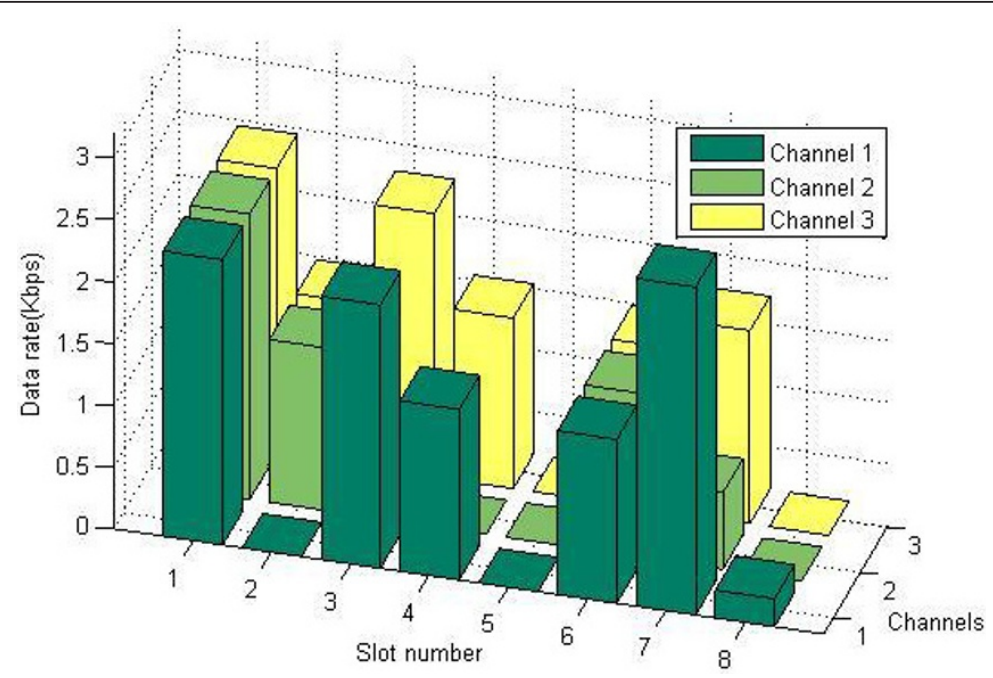

Figure 6 Channel eviction behavior of CR.

The effect of channel eviction on the sum rate is illustrated in Table 2. The sum rate is the sum of data rates achieved on all channels by all CR users. For example, in current case, we consider three channels and the sum rate is equal to the addition of available data rates on all channels. The results show that the optimal sum rate is achieved for the case in which more channels are available for $\mathrm{CR}$ users. The sum rate is maximum during slot number 1 as all channels are being used for $\mathrm{CR}$ transmission. In this case, there is no activity of PU on these channels. The sum rate declines significantly during slot numbers 2,3 , and 4 because of the arrival of PU on the channels 1 and 2 .

The channel sharing pattern of the proposed scheme is also shown in Table 2. We consider six SUs with three transmitters and three receivers. We assume that all SU transmitters are the same power, i.e., $30 \mathrm{~dB}$. The file size and user's ID of SU are managed through a small database. The proposed scheme selects the SUs from FIFO queue and assigns the available channel. The framing is performed before transmission of the data, and the frames of variable size are used depending upon the capacity of channels. For example, channel 2 is assigned to the $\mathrm{SU}$ with ID 2 and $\mathrm{SU}_{2}$ transmits fame of size 2.30 and $1.13 \mathrm{~kb}$ during slot numbers 1 and 2,

Table 2 The sum rate and SUs activity on channels

\begin{tabular}{|c|c|c|c|c|c|c|c|c|}
\hline \multirow[t]{2}{*}{ Channels } & \multicolumn{8}{|c|}{ Slot number } \\
\hline & 1 & 2 & 3 & 4 & 5 & 6 & 7 & 8 \\
\hline 1 & $\mathrm{SU}_{1}$ & $\mathrm{PU}$ & $\mathrm{SU}_{1}$ & $\mathrm{SU}_{1}$ & $\mathrm{PU}$ & $\mathrm{SU}_{1}$ & $\mathrm{SU}_{1}$ & $\mathrm{SU}_{1}$ \\
\hline 2 & $\mathrm{SU}_{2}$ & $\mathrm{SU}_{2}$ & PU & PU & PU & $\mathrm{SU}_{2}$ & $\mathrm{SU}_{2}$ & - \\
\hline 3 & $\mathrm{SU}_{3}$ & $\mathrm{SU}_{3}$ & $\mathrm{SU}_{3}$ & $\mathrm{SU}_{3}$ & PU & $\mathrm{SU}_{3}$ & $\mathrm{SU}_{3}$ & - \\
\hline Sum rate (kbps) & 6.90 & 2.62 & 4.52 & 2.75 & 0 & 3.97 & 4.82 & 0.22 \\
\hline
\end{tabular}

respectively. Hence, OSA maximizes the capacity utilization with variable frame size.

\subsection{Effect of PU arrival on SU's service time and throughput}

Figure $7 \mathrm{a}, \mathrm{b}$ shows the impact of PU arrival activity on the total service time and throughput of SU. In this scenario, we show the outcomes for three different file sizes and five SUs. The SUs have file size of 5,10 , and $15 \mathrm{~kb}$. Therefore; the total file size for each case is 25 , 50 , and $75 \mathrm{~kb}$, respectively.

Figure 7a depicts the delay in the service time because of the arrival rate of PU. In the first case, each SU has a file of $5 \mathrm{~kb}$. All SUs get service in just $5 \mathrm{~s}$. In this case, there is no PU activity on three available channels. The service time increases in linear pattern with the increase of file size. It becomes nearly double (5.9-11 s) when the total file size varies from 25 to $50 \mathrm{~kb}$. Similarly, when we increase the total file size to $75 \mathrm{~kb}$, the required time to serve SUs becomes almost three times (16 s). There is another significant impact on service time because of the increase in the arrival frequency of PU. For the arrival frequency equals to 20 , there is an increase of 110 , 63 , and $43 \%$ in the service time for the file size of 25 , 50 , and $75 \mathrm{~kb}$, respectively.

The effects of arrival activity of PU on small file size are slightly higher since in certain time slot the SU may have less amount of remaining data than the frame size. Figure 7b depicts the degradation in the SU's throughput with the increase in the arrival rate of PU. PU arrival rate is varied from 0 to 1 . The fraction of throughput decreases linearly with the increase in the arrival rate of the PU for larger file size. For small file size, there is a slight variation in the behavior, this is because of the same reason given in Figure 7a. 


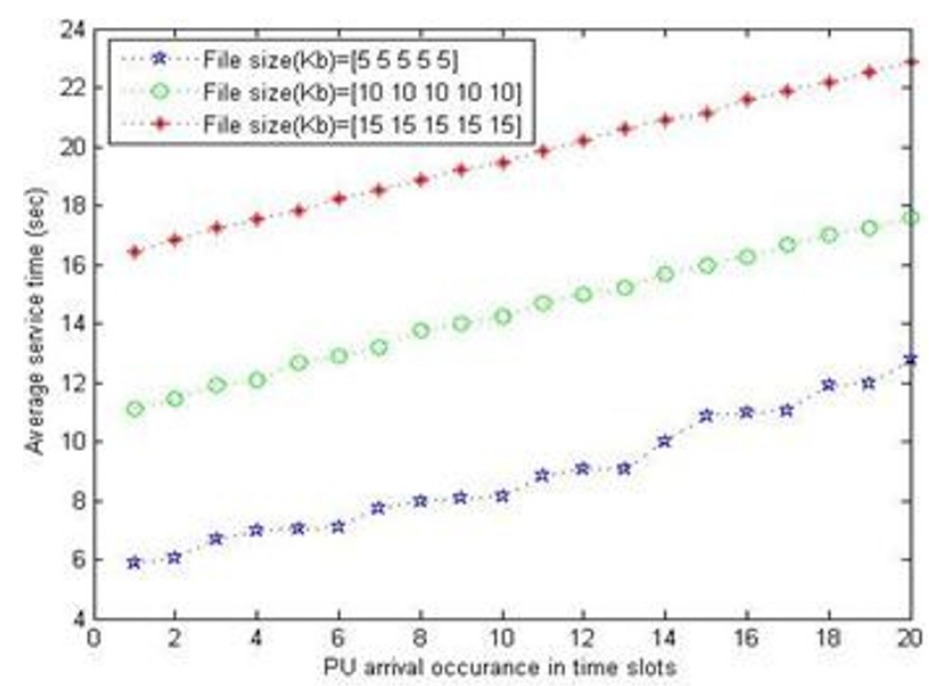

(a)

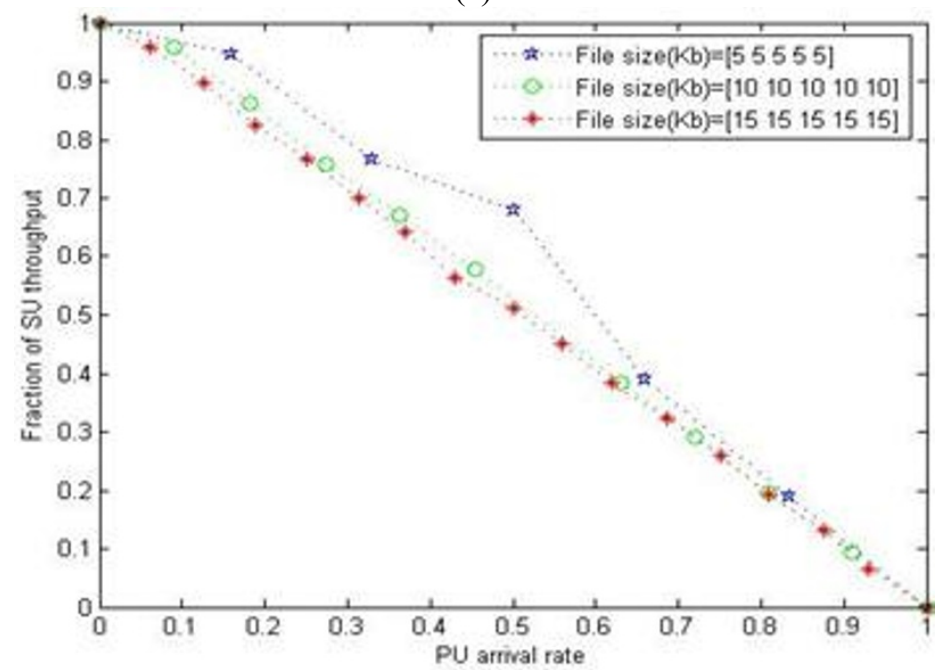

(b)

Figure 7 Impact of PU activity. (a) Effect on average service time; (b) Effect on SU's throughput.

\subsection{Comparison with MMF scheme}

In this section, we compare our proposed FEPO scheme with the MMF scheme in terms of transmission power. The periodic monitoring of PU activity significantly reduces the transmission power.

In this case, we consider two different scenarios: (1) varying file size and (2) varying number of retransmissions for the PU activity illustrated in Figure 4. For simplification, we assume that system consumes $1 \mathrm{~kW}$ to transmit $1 \mathrm{kB}$ data file. In first scenario, the PU arrives six times on channels 1,2 , and 3 , respectively. The FEPO scheme utilizes the PAM block to monitor the arrival activity of PU and does not transmit data when PU arrives on current in use channel and save the significant amount of transmission power. We simulate this scenario for different file sizes varying from 25 to
$125 \mathrm{~kb}$ as shown in Figure 8a. The FEPO transmits the same data file by consuming 10-40\% lower power. For example, to transmit the file size of $25 \mathrm{~kb}$ MMF requires $35 \mathrm{~kW}$ transmission power, whereas the FEPO scheme transmits the same file with transmission power of 25 $\mathrm{kW}$, which is significantly lower power as compared to MMF scheme. Mathematically, the current scenario 1 for a given PU activity (see Figure 4) can be represented as follows.

$$
\begin{aligned}
& \xi(f)=f \\
& \xi(f)=f+(\beta+\gamma)
\end{aligned}
$$

where $\xi(f)$ represents the power requirement for each scheme. The factor $f$ represents the power requirement 


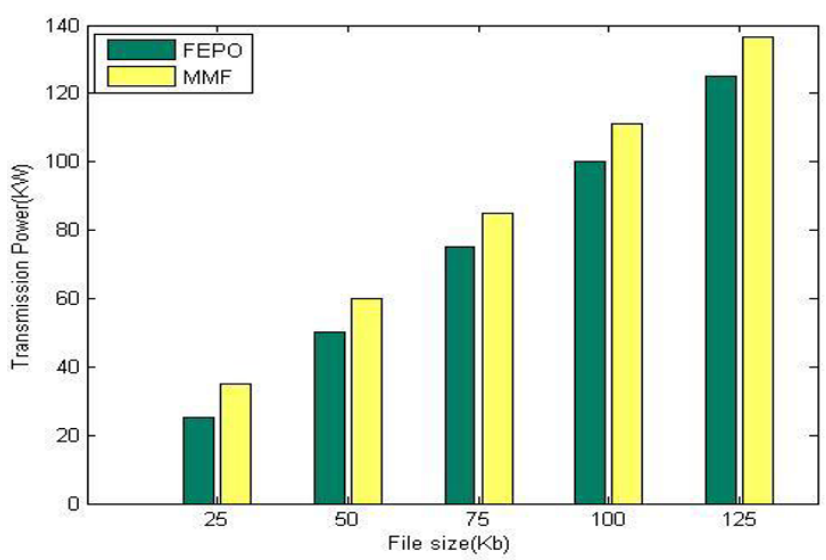

(a)

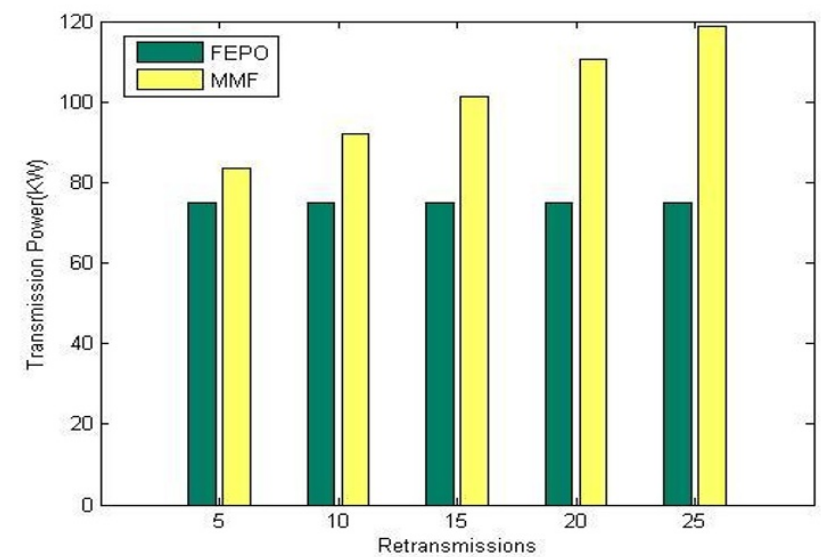

(b)

Figure 8 Comparison between FEPO and MMF scheme in terms of transmission power. (a) Varying file size; (b) Varying number of retransmissions.

of our proposed FEPO scheme. The factor $\beta+\gamma$ indicates the additional power factor for MMF scheme. The $\beta$ factor has a constant value for a given PU activity. The $\gamma$ factor is very small as compared to the $\beta$ factor, $\mathrm{i}$. e., $\gamma<<\beta$. In Figure $8 \mathrm{a}$, the value of $\beta$ factor is 10 and the values of $\gamma$ factor are 1 and 2 for file size of 100 and $125 \mathrm{~kb}$, respectively. The $\gamma$ factor is zero for first three file sizes.

In second case, we show the comparison using file size of $75 \mathrm{~kb}$ and varying the number of retransmissions. Each PU's arrival requires a retransmission if monitoring is not considered properly. The retransmissions are varied from 5 to 25 , and we measure the power consumption for each scheme. It is clear from the simulation results that MMF scheme consumes $11.5-58.5 \%$ extra power for the transmission of same files having size of 25 and $125 \mathrm{~kb}$, respectively. Mathematically, the comparison of scenario 2 can be depicted using the following equations

$$
\begin{aligned}
& \xi(r)=\alpha \\
& \xi(r)=\alpha+k r
\end{aligned}
$$

where $\xi(r)$ represents the power requirement of each scheme. The proposed FEPO scheme requires significantly lower power to transmit a given file. However, the power requirement of MMF scheme increases linearly with the increase in the number of retransmissions. The factor $k$ is the slope factor and $r$ represents the number of retransmissions. In Figure 8b, the value of $\alpha$ factor is $75 \mathrm{~kW}$, the factor $r$ represents the number of retransmissions and $k$ is increasing factor, and its value is 1.82 for $r=20$.

\section{Conclusions}

The spectrum sharing in CRNs is more challenging than the traditional wireless networks. Here, spectrum band varies continuously across the space and time in terms 
of both availability and quality. This varying nature of spectrum demands for a radio environment-aware optimized spectrum allocation mechanism. In this article, we present a spectrum sharing scheme that schedules the sensed spectrum holes among cognitive radio $(\mathrm{CR})$ users by considering the changes occur in the radio environment as well as the PU's activity on current in use channels. In the proposed framework, we assume slotted structure where each CR performs sensing operation at the start of each slot. The CR monitors the in-use channel for PU activity. If the channel is still idle, it will perform the transmission on the same channel otherwise it looks for some other channel for transmission or remains silent during the entire time slot to avoid interference with PU.

We also propose a dynamic framing process at MAC layer, which can form variable size frames depending upon the capacity of available channels. The simulation results show that our proposed scheme outperforms in saving the transmission power while ensuring required throughput and fairness. Moreover, we compare the service time and throughput of CR user against different file sizes. The PU arrival activity on the available channels degrades the performance of the CRN but in our scheme, the periodic monitoring significantly enhances the performance by reducing the number of retransmissions.

\section{Acknowledgements}

This research was supported by the MKE (The Ministry of Knowledge Economy), Korea, under the Convergence-ITRC (Convergence Information Technology Research Center) support program (NIPA-2011-C6150-1101-0003) supervised by the NIPA (National IT Industry Promotion Agency).

\section{Competing interests}

The authors declare that they have no competing interests.

Received: 15 July 2011 Accepted: 13 December 2011

Published: 13 December 2011

\section{References}

1. SJ Kim, Dynamic spectrum allocation with variable bandwidth for cognitive radio systems. Proceedings of the 9th IEEE International Symposium on Communication and Information technology. 106-109 (2009)

2. S Haykin, Cognitive radio: brain-empowered wireless communications. IEEE J Sel Areas Commun. 23(2):201-220 (2005)

3. IF Akyildiz, W Lee, MC Vuran, S Mohanty, NeXt generation/dynamic spectrum access/cognitive radio wireless networks: a survey. J Comput Netw. 50, 2127-2159 (2006). doi:10.1016/j.comnet.2006.05.001

4. RD Yates, CS Raman, NB Mandayam, Fair and efficient scheduling variable rate channels via spectrum server. Proceedings of the IEEE International Conference on Communication. 11, 5246-5251 (2006)

5. A Goldsmith, SA Jafar, I Maric, S Srinivasa, Breaking spectrum gridlock with cognitive radios: an information theoretic perspective. Proc IEEE. 97(5):894-914 (2009)

6. KB Letaief, W Zhang, Cooperative communications for cognitive radio networks. Proc IEE. 97(5):878-893 (2009)

7. S Srinivasa, SA Jafar, How much spectrum sharing is optimal in cognitive radio networks. IEEE Trans Wirel Commun. 7(10):4010-4018 (2008)

8. S Xie, Y Liu, Y Zhang, R Yu, A parallel cooperative spectrum sensing in cognitive radio networks. IEEE Trans Veh Technol. 59(8):4079-4092 (2010)
9. D Cabric, SM Mishra, RW Brodersen, Implementation issues in spectrum sensing for cognitive radios. Proceedings of the Thirty Eighth Asilomar Conferences on Signals, System and Computers. 1, 772-776 (2004)

10. I Katzela, M Naghshineh, Channel assignment schemes for cellular mobile telecommunication systems: a comprehensive survey. IEEE J Pers Commun. 3(3):10-31 (1996). doi:10.1109/98.511762

11. Z Han, Z Ji, KR Liu, Low-complexity OFDMA channel allocation with Nash bargaining solution fairness. Proceedings of the IEEE Global Telecommunications Conference (GLOBECOM '04). 6, 3726-3731 (2004)

12. Q Zhao, BM Sadler, A survey of dynamic spectrum access: signal processing, networking, and regulatory policy. Proceedings of the IEEE International Conference on Acoustic, Speech and Signal Processing. 4(3):1349-1352 (2007)

13. C Cormio, KR Chowdhury, A survey on MAC protocols for cognitive radio networks. J Ad Hoc Netw. 7, 1315-1329 (2009). doi:10.1016/j. adhoc.2009.01.002

14. H Mujeeb, F Aslam, S Aslam, Device-centric spectrum sharing for cognitive radios. Proceeding of the IEEE International conference on networking and Technology. 410-414 (2010)

15. D Cabric, ID O'Donnell, MS-W Chen, RW Brodersen, Spectrum sharing radios. Proceedings of the IEEE Circuits and System Magazine. 6(2):30-45 (2006). doi:10.1109/MCAS.2006.1648988

16. W Hu, M Gerla, GA Vlantis, GJ Pottie, Efficient, flexible and scalable internetwork spectrum sharing and communications in cognitive IEEE 802.22 networks. Proceedings of the IEEE Conference on Cognitive Radio and Advanced Spectrum Management (COGART '08). 1-5 (2008)

17. S Debroy, M Chatterjee, Intra-cell channel allocation scheme in IEEE 802.22 networks. Proceeding of the IEEE Consumer Communication and Networking Conference (CCNC '10). 1-6 (2010)

18. V Brik, E Rozner, S Banerjee, P Bahl, DSAP: a protocol for coordinated spectrum access. Proceedings of the First IEEE Symposium on New Frontiers in Dynamic Spectrum Access Networks. 611-614 (2005)

19. XL Huang, B Bensaou, On max-min fairness and scheduling in wireless adhoc networks: analytical framework and implementation. Proceedings of the Second ACM International Symposium on Mobile and Ad-hoc Networking and Computing. 221-231 (2001)

20. T Nandagopal, T Kim, X Gao, V Bharghavan, Achieving mac layer fairness in wireless packet networks. Proceedings of the sixth Annual International Conference on Mobile Computing and Networking. 87-98 (2000)

21. H Luo, S Lu, V Bharghavan, A new model for packet scheduling in multihop wireless networks. Proceedings of the sixth Annual International Conference on Mobile Computing and Networking. 76-86 (2000)

22. $\vee$ Asghari, S Aissa, Adaptive rate and power transmission in spectrum sharing systems. IEEE Trans Wirel Commun. 9, 1-5 (2010)

23. VK Tumuluru, P Wang, D Niyato, Channel status prediction for cognitive radio networks. J Wirel Commun Mob Comput (WCMC). 10, 1-13 (2010). doi:10.1002/wcm.909

24. U Yuan, RC Grammenos, Y Yang, W Wang, Performance analysis of selective opportunistic spectrum success with traffic prediction. IEEE Trans Veh Technol. 59(4):1949-1959 (2010)

25. M Hoyhtya, S Pollin, A Mammela, Classification-based predictive channel selection for cognitive radios. Proceeding of the IEEE International Conference on Communication (ICC '10). 1-6 (2010)

26. KW Sung, S-L Kim, J Zander, Temporal spectrum sharing based on primary user activity prediction. IEEE Trans Wirel Commun. 9(12):3848-3855 (2010)

27. Q Zhao, K Lin, L Tong, BM Sadler, Opportunistic spectrum access via periodic sensing. IEEE Trans Signal Process. 56(2):33-37 (2008)

doi:10.1186/1687-1499-2011-201

Cite this article as: Aslam and Lee: Fair, efficient, and power-optimized spectrum sharing scheme for cognitive radio networks. EURASIP Journal on Wireless Communications and Networking 2011 2011:201. 